\title{
Inserción Profesional Docente: problemas y éxitos de los profesores principiantes*
}

\author{
Inserting Professional Teaching: Problems and successes of beginning teachers \\ Inserção Profissional de Ensino: Problemas e sucessos de professores iniciantes
}

\begin{abstract}
María Cristina Solís Zañartu, ${ }^{a}$ Claudio Núñez Vega ${ }^{b}$ Inés Contreras Valenzuela, ${ }^{c}$ Nelson Vásquez Larad ${ }^{\text {, Sylvia Ritterhaussen Klaunning }}{ }^{\text {. }}$
\end{abstract}

aPontificia Universidad Católica de Chile

Telf.: (56-2) 23545382, Correo electrónico: csolisz@uc.cl

'Pontificia Universidad Católica de Chile

Telf.: (56-2) 23545359, Correo electrónico: cnunezv@uc.cl

'Pontificia Universidad Católica de Chile

Telf.: (56-2) 23545386, Correo electrónico: icontrev@uc.cl

dPontificia Universidad Católica de Valparaíso

Telf.: (56-32) 2273211, Correo electrónico: nvasquez@ucv.cl

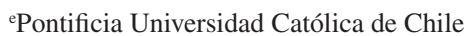

Telf.: (56)222117931. Correo electrónico: sritters@uc.cl

\begin{abstract}
RESUMEN
Este estudio presenta los resultados del análisis de los problemas y éxitos en el desempeño profesional de profesores de educación básica y educación media durante los primeros años de inserción laboral, egresados de seis universidades chilenas. Los resultados describen las dimensiones del desarrollo profesional que son percibidas como dificultades y éxitos por parte de los profesores que se insertan al mundo laboral de la docencia, así como la visión de directivos de los colegios. Basado en los hallazgos se concluyó que hay dimensiones del desempeño que constituyen fortalezas, en cambio otras vinculadas con Manejo de recursos de aprendizaje diferenciados, Interacciones con otros actores, Inserción institucional, Realización de tareas simultáneas y Manejo de problemas sociales son percibidas como dificultades. Además, se encontró que los éxitos obtenidos evolucionan a través de los primeros años de inserción.
\end{abstract}

Palabras clave: profesores principiantes, inserción profesional, dificultades y éxitos.

\section{ABSTRACT}

This study presents the results of an analysis of the problems and successes in the professional performance of teachers of basic and secondary education in the early years of employment, graduated from six Chilean universities. The results describe the dimensions of professional development that are perceived as challenges and successes by teachers that are inserted into the world of teaching work, as well as the vision of school managers. Based on the findings it was concluded that there are dimensions of performance that are strengths, however other related with differentiated learning resources management, interactions with other actors, institutional insertion, performing simultaneous tasks and handling social problems are perceived as difficulties. Furthermore, it was found that the successes evolve through the early years of insertion.

Key words: beginning teachers, employability, difficulties and successes.

\footnotetext{
Este estudio forma parte de la investigación FONDECYT 1131091.
} 


\section{ANTECEDENTES}

Este estudio es parte de una investigación (Fondecyt 1131091) cuyo propósito es "Establecer las condiciones de inserción profesional de los egresados de las carreras de pedagogía media y básica a partir de las relaciones entre las dificultades y niveles de desempeño de los profesores novatos, las herramientas con que cuentan para resolver dichos problemas y los sistemas de apoyo desde las instituciones educativas.". La fuente de información estuvo constituida por profesores novatos de seis instituciones formadoras de profesores en Chile, tanto de educación básica como de educación media, directivos de dichas carreras y por directores de los establecimientos en los cuales esos profesores novatos se desempeñaban.

Esta investigación implica la realización de dos tipos de estudio. Uno de tipo transeccional, que busca conocer la opinión de los profesores novatos con respecto a las dificultades y éxitos durante sus tres primeros años de inserción laboral y la opinión de los directivos de establecimientos en que se desempeñan, con respecto a los apoyos que brindan a los profesores novatos en su proceso de inserción laboral. El otro es un estudio longitudinal que busca indagar respecto de la evolución de los profesores novatos en su desempeño en aula en los primeros tres años de inserción profesional y su percepción de las dificultades que enfrentan a medida que se desarrollan profesionalmente.

Los resultados que se presentan en este artículo corresponden al estudio transeccional y permiten conocer las dificultades y éxitos percibidos por los profesores egresados de pedagogía media y básica de seis universidades chilenas, que están en sus tres primeros años de inserción a la vida laboral y que se desempeñan en establecimientos ubicados en diferentes regiones del país.

\section{MARCO REFERENCIAL}

El inicio de la profesión docente sin duda implica un desafío para los profesores jóvenes; ellos transitan desde el rol de estudiantes al de profesores, dejan una institución que les es conocida, en la que tienen pares con los cuales han compartido algunos años y con los que se sienten apoyados en su acción, para llegar a una institución cuya cultura desconocen, en la cual deben asumir un nuevo rol y en la que se sienten solos. Entonces, al insertarse a la vida profesional, los profesores principiantes suelen enfrentarse a una serie de problemáticas, algunas derivadas de las nuevas relaciones interpersonales que deben establecer, del escaso conocimiento de la cultura escolar o de las múltiples tareas que deben asumir en forma simultánea, esto sumado a que deben asumir su rol de enseñantes para el cual fueron formados.

La problemática de la inserción profesional docente ha sido abordada en diferentes estudios nacionales e internacionales, algunos de los cuales se refieren a esta etapa como una más en el desarrollo profesional aunque con características propias, que cambian a medida que el nuevo profesional va adquiriendo conocimientos de su disciplina pedagógica y de los contextos en los que se desenvuelve. Otros la consideran como un período de transición entre la formación y la vida profesional, sin embargo todos coinciden en que hay un conjunto de dificultades que el profesor principiante debe sortear durante los primeros años de inserción. Por ejemplo, Marcelo en su libro Profesorado Principiante 
(Marcelo, 2008) señala que la inserción profesional es el período de tiempo en el cual los profesores transitan desde estudiantes a maestros, además hace referencia al autor holandés Vonk, quien señala que en este período hay una transición entre profesor en formación y profesional autónomo, etapas que forman parte del continuo del desarrollo profesional.

La experiencia del profesor novato en sus primeros años dependerá de sus capacidades para conocer la institución y sortear las dificultades con que se encuentra, independientemente de la preparación que le ha entregado la institución formadora y de los apoyos que recibe de la institución en la que trabaja. Diversas investigaciones han puesto en evidencia como el profesor principiante se enfrenta a la realidad laboral casi en una situación de shock que lo hace vivir una serie de dificultades que debe enfrentar y resolver con sus propios recursos o con apoyos externos (Alliaud, 2004; Marcelo, 2008 y 2009; Veenman, 1984). Sin embargo, estas situaciones que en un comienzo parecen difíciles y casi imposibles de solucionar, cimentarán parte del gran camino que se debe recorrer para llegar a ser un profesor experto.

Otro autor (Feiman-Nemser, 2000) indica que los profesores en este período son tanto aprendices como enseñantes, por una parte deben enseñar pero también tienen que aprender a enseñar. En este contexto de aprendizaje, los profesores principiantes tienen que conocer a sus estudiantes, a la institución, a las familias, el currículo escolar, todo esto sumado a que deben enseñar y asumir la responsabilidad del aprendizaje de sus estudiantes. Es un período que provoca tensiones, pero también desafíos y aprendizajes porque se requiere conocer una institución y a un grupo profesional desconocido que tiene una cultura particular, porque se debe adquirir conocimiento profesional y además mantener un equilibrio personal. Se podría decir que en este período el principiante se convierte en un equilibrista que debe sopesar y balancear varias situaciones en forma armónica, de manera tal que en su conjunto contribuyan a su desarrollo profesional.

En el texto ya mencionado referido a profesores principiantes (Marcelo, 2009), hace referencia a una serie de investigaciones que han estudiado la problemática de los profesores principiantes, por ejemplo las realizadas por Veeman y Vali entre otras, quienes comparten la idea de que empezar a trabajar es una experiencia dura, en la que los novatos se enfrentan a una serie de problemas como son la interiorización de normas de la institución, el trabajo con sus pares, la relación que deben establecer con apoderados o el comportamiento de los alumnos, problemas que en ocasiones son causa de deserción o abandono de la docencia en los primeros años. En Estados Unidos, Cochran-Smith (2004) plantea que para evitar estas tensiones los principiantes no deben trabajar en forma aislada, sino que necesitan ciertas condiciones de la escuela como es el trabajo con pares y la creación de comunidades de aprendizaje.

En Chile (Avalos et al., 2004) en una revisión bibliográfica relacionada con profesores principiantes cita varios estudios que se refieren a los problemas que viven los profesores al inicio de su vida profesional, por ejemplo la falta de tiempo para enseñar, el elevado número de alumnos por clase, la mala calidad de la infraestructura y la escasez de material (Veeman, 1984; Marcelo, 1993). Los autores también mencionan otro estudio (Huberman, Gonauer y Marti, 1993) en que los problemas se categorizan en a) problemas personales no vinculados a la enseñanza, de índole económico o familiar entre otros y b) pedagógicos, por ejemplo las relaciones con los alumnos, el dominio de los conocimientos de lo que deben enseñar, las relaciones con sus colegas, las relaciones con la institución escolar, además de su preparación profesional.

En Uruguay, (Rodríguez, 2007) concluyó que la realidad de los profesores novatos no es tan distinta a la que ocurre en otros países. Menciona que una dificultad relevante 
del primer año es la coacción de las autoridades de los establecimientos escolares por lograr determinados propósitos, lo que afecta la capacidad de los profesores novatos para tomar decisiones. También releva que no hay pautas, orientaciones y estructuras para la coordinación de las actividades que hace este profesor. En muchos casos existiría un choque con el aula y la realidad escolar, porque no hay suficientes espacios para la innovación pedagógica que los profesores principiantes quisieran realizar.

En otra investigación acerca de las debilidades de los profesores principiantes norteamericanos (Thompson, 2007), las dificultades se sistematizaron en tres grupos. El primero es el ámbito personal, cuyas dificultades estaban relacionadas con el manejo del tiempo, las interrelaciones personales, el asumir distintas labores en forma simultánea, el stress y nerviosismo frente a la observación, entre otros aspectos. El segundo es el ámbito de la planificación y manejo de la clase, en el cual las dificultades se relacionan con el desconocimiento de lineamientos curriculares, apresuramiento por cubrir las materias escolares en vez de preocuparse del aprendizaje, insuficientes oportunidades de aprendizaje o la no consideración de los conocimientos previos y de los estilos de aprendizaje. El tercer ámbito está referido a la evaluación, las dificultades se resumen en escasez de evaluaciones formativas, diseño y construcción de instrumentos evaluativos adecuados para evaluar lo que efectivamente se enseñó y herramientas para evaluar el progreso de los estudiantes.

En otro estudio en Canadá en el año 2010, las investigadoras St. Vicent y Gervais al preguntarse si un profesor novicio está preparado para afrontar problemas éticos en la escuela concluyen que, cuando ellos deben analizar un problema educacional que aborda aspectos éticos, evocan más sus preocupaciones relacionadas con la inserción profesional que con las dimensiones del problema ético propiamente tal. Por ejemplo, las dificultades que tienen con sus colegas o con los padres o con los alumnos debido a las diferencias culturales no son abordadas desde sus implicancias éticas, sino que afloran otras preocupaciones como el foco de su quehacer. Así también, sus propias problemáticas como profesionales no les permiten analizar los elementos que constituyen ese problema ético que se les presenta, reconociendo además que aún no tienen experiencia para abordarlo porque su credibilidad respecto de sus propuestas y acciones puede ser frágil.

Estudios en Chile (Avalos et al., 2004) confirman que los docentes principiantes tienen las mismas dificultades que los profesores novicios de otros países. Sin embargo, señalan que además los chilenos no reciben ayuda formal para abordar y dar solución a los problemas, y en general, los novatos adquieren experiencia y resuelven sus dificultades a través de ensayo y error. Por su parte, en un estudio relacionado con profesores principiantes de Educación Básica (Cisternas, 2011), señala que las dificultades que ellos enfrentan muestran diferencias respecto de su naturaleza, nivel de complejidad y profundidad dependiendo de tres factores: a) las oportunidades que la formación inicial proporcionó para problematizar la realidad, b) las características de la institución escolar en la que se insertan y c) las condiciones personales del profesor principiante.

En un artículo relacionado con programas de inducción (Boerr, 2014) plantea que adquirir conocimiento profesional es una tarea compleja porque se trata principalmente de integrar los conocimientos teóricos a la práctica y responder a la demanda de soluciones rápidas, teniendo presente el conocimiento del contexto, la organización del tiempo, la conducción de los estudiantes y sus conflictos y el manejo de instrumentos curriculares.

Resultados preliminares de una reciente investigación (Contreras et al., 2013), que incluye entrevistas a profesores novatos de enseñanza media egresados de dos universidades 
chilenas, en relación con su primer año de experiencia laboral, permitieron detectar un conjunto de necesidades y dificultades que vivieron estos profesores al insertarse en el aula y en los establecimientos escolares. En términos generales, se establece que el factor tiempo es el problema más evidente, tienen mucho trabajo y poco tiempo para cumplir con todas las tareas demandadas y también deben asumir una multiplicidad de responsabilidades que los estresa, además de no sentirse preparados para asumirlas.

A modo de síntesis, se puede señalar que las investigaciones nacionales e internacionales en los últimos treinta años son coincidentes en plantear que la inserción profesional docente es un período muy difícil de abordar debido a la multiplicidad de problemáticas que afectan a los docentes principiantes. Dada esta realidad pareciera importante generar mecanismos para que las instituciones formadoras y los establecimientos educacionales propongan acciones para facilitar la inserción profesional y contribuir al desarrollo profesional de los docentes que se integran al mundo laboral.

\section{METODOLOGÍA}

Para indagar en las dificultades y éxitos de los profesores principiantes en su experiencia de inserción profesional en establecimientos educacionales, se aplicó un cuestionario que contenía tres apartados: Desempeños profesionales, Condiciones de trabajo para el desempeño profesional, Apoyos para la inserción profesional.

En relación con el apartado de desempeños profesionales de los cuales da cuenta este artículo, el cuestionario estuvo constituido por 19 afirmaciones y una escala de respuesta con cuatro categorías, las que se redujeron a dos para efectos de análisis. Las afirmaciones respondían a las siguientes dimensiones correspondientes al quehacer docente: planificación de la enseñanza y de la evaluación, manejo de clima, manejo de actividades y recursos de aprendizaje, uso de recursos de aprendizaje diferenciados, interacciones con otros actores, inserción institucional, realización de tareas simultáneas y manejo de problemáticas sociales.

Además el cuestionario contenía dos preguntas abiertas; en la primera se pedía a los profesores novatos mencionar la principal dificultad a la que se había enfrentado en los tres primeros años de trabajo y la forma solucionarla, en tanto que en la segunda se les pidió mencionar el principal éxito profesional en sus años de trabajo.

El instrumento fue aplicado a 187 profesores novatos, de los cuales 88 son profesores de educación básica (47\%) y 99 (53\%) de educación media, egresados en 6 instituciones formadoras de profesores en Chile y que se desempeñan en distintos tipos de establecimientos educacionales de dependencia municipal, particular y particular subvencionado, ubicados en cuatro regiones del país.

Del total de profesores novatos, 91 (49\%) estaban en su primer año de inserción, 42 (22\%) en segundo año y 54 (29\%) en tercer año de trabajo.

Para seleccionar la muestra se contó con bases de datos de los egresados de cada universidad en los tres últimos años. Estos profesores fueron invitados a participar en la investigación, siendo requisito fundamental el que estuviesen trabajando en un establecimiento educacional. Los profesores novatos que respondieron afirmativamente fueron contactados telefónicamente para conocer los alcances de la investigación y finalmente acordar la formalización de su participación. 
Además, se diseñó un instrumento con preguntas equivalentes al anterior para conocer la opinión de los directivos de los establecimientos educativos en el cual trabajaban los profesores participantes en la investigación. Este instrumento se aplicó a 84 directivos y recoge su opinión acerca de los apoyos dados en dichos establecimientos respecto de las diferentes dimensiones de desempeño profesional.

El análisis de las respuestas cerradas permitió determinar los desempeños profesionales, en término de dificultades para cada una de las dimensiones, además se comparó si había diferencias entre los diferentes años de ejercicio profesional. Para las respuestas abiertas se hizo un análisis cualitativo para establecer si los tópicos acerca de problemas y éxitos cambiaban durante los tres años de ejercicio docente.

\section{RESULTADOS}

A partir de la aplicación del Cuestionario Opinión de Profesores Novatos, los resultados evidencian las declaraciones de los profesores respecto de las dificultades y éxitos en su desempeño, en sus tres primeros años de ejercicio profesional.

Respecto de las dificultades, en cuanto a la dimensión "Planificación de la enseñanza y evaluación", los profesores novatos manifiestan no tener dificultades para tomar decisiones en aspectos pedagógicos relativos a la planificación de la enseñanza y planificación de la evaluación en primer año (85\%), segundo año (88\%) y tercer año (83\%), lo cual estaría evidenciando que en sus procesos formativos se ha estimulado el desarrollo de competencias para desempeñarse en dichos procesos como son la toma de decisiones sobre la planificación de la enseñanza y de la evaluación. Sin embargo, estas opiniones de los profesores novatos contrastan con la opinión de los directivos de establecimientos, quienes manifiestan brindar instancias de apoyo (92\%) a los profesores que se integran a sus establecimientos en las temáticas de planificación de la enseñanza y evaluación, lo cual podría ser un indicador de que los profesores requieren de apoyo. Al analizar las opiniones de los profesores en los tres primeros años en los diferentes componentes de la dimensión, no se observan diferencias significativas.

Respecto de la dimensión "Manejo clima" los profesores novatos señalan que no tendrían dificultades para conducir procesos de enseñanza durante su primer año de inserción (58\%), segundo año (74\%) y tercer año (50\%). Cabe destacar que las opiniones de los profesores de primer y tercer año evidencian que esa tendencia sería menor principalmente en lo relativo al manejo de estrategias para captar la atención de los alumnos y estrategias para involucrar a los alumnos en el aprendizaje. Al analizar las opiniones de los profesores en los distintos años de inserción profesional encontramos que sólo existen diferencias significativas en el Manejo de estrategias para captar la atención de los alumnos $\chi^{2}(2, \mathrm{~N}=$ $187)=8,419, \mathrm{p}=.015$ ). Por su parte, el $82 \%$ de los directivos de colegios plantea que los profesores en sus primeros años reciben apoyo en temáticas vinculadas a manejo de clima.

En la dimensión "Manejo de actividades y recursos de aprendizaje" los profesores principiantes de primer año (73\%), segundo año (63\%) y tercer año (63\%) expresan mayoritariamente que no han tenido dificultades en acciones relativas a la conducción de actividades de aprendizaje variadas y de diferente nivel de complejidad, uso de recursos didácticos variados e instrumentos de evaluación, así como manejo de contenidos. En este caso no se encuentran diferencias significativas en los diferentes indicadores de la 
dimensión entre los tres primeros años de inserción profesional. No obstante, los directores de colegios señalan que en sus establecimientos proporcionan apoyo $(78 \%)$ en temáticas vinculadas con esta dimensión.

La dimensión "Manejo de recursos de aprendizaje diferenciados" es percibida por los profesores novatos como una dificultad en primer año (47\%), segundo año (49\%) y tercer año $(56 \%)$. Ellos manifiestan tener problemas para usar recursos didácticos y evaluativos diferenciados, acordes a las características de sus estudiantes. Al analizar las opiniones de los profesores novatos llama la atención que a lo largo de los tres años aumenta la proporción de profesores que perciben tener dificultades en esta dimensión, sin embargo a nivel estadístico no se encuentran diferencias significativas. Las opiniones de los profesores novatos son concordantes con las de los directivos de colegios (70\%) quienes declaran que proporcionan apoyos a sus profesores en aspectos vinculados a uso de recursos didácticos y evaluativos.

En la dimensión "Interacciones con otros actores", los profesores principiantes declaran tener dificultades en su primer año (43\%), segundo año (31\%) y tercer año (58\%) tanto en lo relativo al trabajo con apoderados como en la interacción con colegas y directivos de los colegios en que se desempeñan. En esta dimensión sólo se encuentran diferencias significativas en cuanto al indicador Interacción profesional con colegas y directivos $\chi^{2}$ $(2, \mathrm{~N}=186)=7,416, \mathrm{p}=.025)$ al contrastar las opiniones entre los tres primeros años de inserción. Por su parte los directivos de colegios (77\%) plantean que entregan a los profesores novatos de sus colegios apoyos para facilitar la interacción con diversos actores de la institución educativa.

En la dimensión "Inserción institucional" los profesores principiantes de primer año (48\%), segundo año (48\%) y tercer año (57\%) plantean tener dificultades para insertarse como profesor en las instituciones y ejecutar tareas variadas. Sin embargo no se encuentran diferencias significativas entre los profesores de los tres primeros años de inserción profesional. Por su parte los directivos (74\%) señalan que dan apoyo a los profesores en los colegios, en aspectos relativos a la inserción como profesionales.

La dimensión "Realización de tareas simultáneas" es percibida por los profesores novatos como una dificultad en primer año (52\%), segundo año (43\%) y tercer año (56\%). Al analizar los resultados de los tres primeros años, no se encuentran diferencias significativas en los diferentes indicadores de la dimensión. Los directivos (70\%) plantean que brindan apoyo en esta dimensión a sus profesores que se insertan en los colegios.

En la dimensión "Manejo de problemas sociales" los profesores principiantes manifiestan tener dificultades en primer año (53\%), segundo año (57\%) y tercer año (56\%) para manejar problemáticas sociales y de alto riesgo. En esta dimensión no se encuentran diferencias significativas en cada indicador en los primeros tres años de inserción laboral. Los directivos (60\%) señalan que en los colegios se otorga apoyo durante la inserción de profesores al establecimiento en temáticas relativas a la dimensión.

Del análisis de las respuestas a las preguntas abiertas en relación con las principales dificultades mencionadas por los profesores principiantes en su primer año de trabajo, ellos manifiestan limitaciones referidas a problemas administrativos del quehacer profesional, por ejemplo:

"Poder cumplir con todo lo administrativo sobre todo con el llenado del libro de clases durante las clases". "Falta de conocimiento respecto del uso y registro en el libro de clases". 
Además problemas vinculados con la conducción de grupos:

"Trabajar con cursos de $5^{\circ}$ y $6^{\circ}$ básico por la falta de disciplina". "Lograr imponer o conservar normas de convivencia con los estudiantes".

Los profesores de segundo año focalizan sus dificultades desde la enseñanza y las metodologías propias de la disciplina y del centro educativo:

"Manejo de la metodología de la disciplina, ya que funcionan con un programa propio". "Inexperiencia en el quehacer pedagógico". "Lidiar contra la experiencia de los docentes, introduciendo nuevas metodologías.". "Conducción de actividades distintas y niveles de complejidad". "Adecuarme a la modalidad de trabajo PAC y uso de la plataforma digital".

En tanto que los profesores de tercer año vuelven a referirse a dificultades en el manejo conductual del grupo de estudiantes:

"La dificultad que me enfrenté fue cuando los alumnos se pusieron de acuerdo a no dar una prueba, porque a sus compañeros que no sabían les iba a ir mal y por apoyarlos sus compañeros se cuadraron con ellos". "Manejo de grupo y resolución de conflictos".

También estos profesores agregan otras respuestas referidas a dificultades que emanan de la organización escolar, por ejemplo:

"No conocer procedimientos y protocolos a seguir para una gran diversidad de situaciones".

"Realizar mucho trabajo administrativo y extra aula". "Organizar/coordinar uno o más eventos anuales planificados por el establecimiento (ej. Día del apoderado) y cumplir tareas específicas en celebraciones". "Comprender la organización y PEI del establecimiento". "Adaptación a los conceptos de formación religiosa y valórica”.

En relación con los éxitos percibidos por los profesores, llama la atención que los profesores principiantes de primer año no responden a la pregunta relacionada con éxitos, de esto se podría inferir que no reconocen éxitos al estar en un período casi de sobrevivencia en la institución escolar, lo que se demuestra en la variedad de respuestas acerca de las dificultades, las que están referidas a tópicos variados.

En cambio algunos profesores de segundo año si reconocen éxitos, aunque relacionados con tópicos diversos:

a) su propio desarrollo profesional:

"Estar en proceso de finalización de un postítulo en matemática, gracias a esto me encuentro en este lugar de trabajo y ejerciendo talleres de matemática a dos Iros medios del colegio". "Mayor proyección laboral, asumiendo la jefatura de un grupo curso".

b) asumir nuevas tareas en el centro educativo:

"Asumir la jefatura del curso después de haber llegado como asistente de aula". "Tener el desafio de una jefatura y que apoderados y directivos reconozcan el trabajo que uno hace con sus alumnos". 
c) con el manejo del grupo:

"Lograr dominar el curso, en comparación a como llegué a principio de año, tomando en cuenta sus características sociales".

d) con aprendizajes de los alumnos:

"El aprendizaje de mis alumnos manifestado en la creación de proyectos, la creatividad en trabajos y evaluaciones diversas". "Lograr buenos resultados en los estudiantes, a pesar de las condiciones estructurales del establecimiento".

Finalmente, la mayoría de los profesores de tercer año reconoce éxitos y estos se asocian principalmente al aprendizaje de sus alumnos, como se aprecia en las siguientes respuestas:

"Mejores clases". "Buenos resultados de aprendizaje en los alumnos". "Premio mejor alumno en su categoría $2^{\circ}$ medio en olimpiadas química y encargada academia científica". "Pasar el $80 \%$ del contenido". "Pensar histórica e historiográficamente subiendo resultados cualitativos y cuantitativos". "Trabajo en conjunto con Educadora Diferencial y utilización de estrategias para facilitar la comprensión lectora y el gusto por la investigación científica". "Generar el gusto por la lectura autónoma e independiente". "Aplicación de un plan de evaluación sistemática para los alumnos, usando diversas evidencias (pruebas, actividades de proceso, cuaderno, participación, etc.) que ha orientado a los estudiantes para mejorar su actitud y rendimiento".

También se aprecian respuestas relacionadas con la valoración de su propia gestión; por ejemplo:

"Consolidar mi inserción en la identidad del establecimiento". "Enseñar a leer y a escribir a mis alumnos". "Lograr trabajar con estudiantes diferenciados (problemas motores, con lenguaje y escritura, problemas sociales y psicológicos). "Lograr con éxito la tutoría de cursos y asumir nuevos desafios".

\section{CONCLUSIONES Y PROYECCIONES}

El análisis de los resultados de este estudio ha permitido reconocer las dificultades y éxitos percibidos por profesores principiantes de pedagogía básica y media en sus tres primeros años de ejercicio profesional, además de los apoyos que los directivos de colegios ofrecen a sus profesores.

Las dificultades que se destacan están vinculadas con las siguientes dimensiones del desempeño profesional: Manejo de recursos de aprendizaje diferenciados, Interacciones con otros actores, Inserción institucional, Realización de tareas simultáneas y Manejo de problemas sociales.

En relación con la dimensión "manejo de recursos de aprendizaje diferenciados" los profesores perciben que no han logrado ser competentes en el uso específico de recursos didácticos para el aprendizaje de alumnos heterogéneos, así como también se sienten que no manejan en sus propuestas evaluativas recursos que les permitan diversificar las formas de recoger evidencias de acuerdo con las características de sus alumnos, aspecto similar a los encontrados en otro estudio (Thompson, 2007). 
Las dificultades en la dimensión "interacción con otros actores" se manifiestan en la falta de estrategias para el trabajo con los apoderados y las formas de interacción profesional con colegas y directivos, lo que concuerda con hallazgos de otras investigaciones acerca de profesores principiantes (Huberman, Gonauer y Marti, 1993) y se ilustra con el siguiente comentario "La mayor dificultad ha sido el tratar con apoderados y el manejo de situaciones en las reuniones y entrevistas con estos".

Las dificultades referidas a la dimensión "inserción institucional" se vinculan con la falta de estrategias para insertarse como profesionales en los colegios y sentirse valorados como tales, lo que se refleja en el siguiente comentario: “Ser muy joven para hacer clases (24 años), la juventud de un recién titulado se toma como sinónimo de inexperiencia, poco dominio de grupo y disciplina, imagen que los profesores y apoderados expresan". Otra dificultad es la incapacidad de los profesores principiantes para ejecutar tareas de manera simultánea en el aula, por ejemplo hacer la clase y en forma simultanea atender el comportamiento disruptivo, completar el libro de clases y otras tareas administrativas. Además, manifiestan el agobio que les significa asumir una diversidad de tareas a nivel del colegio, que expresan como

"Coberturas curriculares mensuales, preparación de actos y actividades del establecimiento, trabajar en horarios fuera de la carga horaria y realizar actividades de otras asignaturas". "Entrevista con apoderados de la jefatura de curso y reuniones, elaboración de informes de alumnos con alguna dificultad y condicionalidad de alumnos, preparación de actividades extraprogramáticas (actos y convivencias) y reuniones con directivos por contingencias". "Talleres de reforzamiento Simce, concursos comunales y del establecimiento, atención de apoderados y asistencia a cursos de especialización dentro de la jornada".

Finalmente, respecto de la problemática asociada a la dimensión "Manejo de problemas sociales", destacan la incapacidad para manejar problemáticas sociales contingentes y en mayor medida el tener estrategias para manejar situaciones de alto riesgo, por ejemplo "Tengo dificultad para trabajar con los alumnos en situación de consumo de drogas y violencia". "Tratar problemas de conducta en el aula asociados a situaciones de índole familiar que afectan a los alumnos".

A modo de síntesis, es posible afirmar que las dificultades en diversos ámbitos del desempeño profesional de los profesores, egresados de instituciones formadoras chilenas, permiten confirmar los hallazgos de otras investigaciones, tanto nacionales como internacionales, respecto de las áreas problemáticas y las dificultades a las que se enfrentan los profesores principiantes al inicio de su vida profesional docente.

En relación con los éxitos, del análisis de las respuestas de los profesores novatos se infiere que hay una evolución en la percepción de éxitos; desde no reconocer éxitos en primer año, luego relacionar el éxito con aspectos asociados al manejo conductual y a su desarrollo profesional en la institución, en tanto que en tercer año claramente se hace mayor referencia al éxito del profesor asociado al aprendizaje de sus alumnos. Estos resultados son coherentes con la revisión realizada por investigadores chilenos (Avalos et al., 2004) quienes citan a varios autores que coinciden en una la evolución de las preocupaciones de los profesores novatos; el inicio de la vida profesional se distingue por acciones de ensayo y error, destinadas a enfrentar las demandas emergentes del trabajo en el aula, en tanto que más adelante se fortalecen las asociaciones entre práctica de enseñanza, aprendizaje académico y las características y necesidades de los alumnos. Las preocupaciones o problemas de los profesores principiantes evolucionan desde preocupaciones acerca de sí 
mismo hacia preocupaciones referidas a las tareas/situaciones y finalmente se cuestionan su impacto sobre los alumnos. Esto se vincula con la percepción que tienen los profesores novatos respecto de sus éxitos.

A partir de las opiniones de los directivos de los colegios pareciera que las acciones de apoyo que dicen entregar a los profesores novatos no están vinculadas con las reales necesidades que estos manifiestan. Es más, algunos directivos comentaron que les resultó interesante la tarea, porque les había permitido darse cuenta que existen otros ámbitos en los cuales podrían apoyar a los profesores que inician su vida laboral. Por otra parte, parece evidenciarse que en los establecimientos educacionales no hay lineamientos ni acciones sistemáticas tendientes a apoyar el proceso de inserción y desarrollo profesional docente.

A partir de este estudio se puede proyectar la necesidad de indagar en profundidad acerca de los éxitos de los profesores en sus primeros años de desempeño docente, de manera de establecer áreas para las cuales las instituciones formadoras proporcionan más experiencias de aprendizaje que favorecen el desempeño profesional futuro y, por tanto, las fortalezas en la formación. Además, de detectar las áreas en que no hay éxito y por tanto requieren apoyo tanto de la universidad como de los colegios.

\section{REFERENCIAS BIBLIOGRÁFICAS}

Alliaud, A. (2004). La experiencia escolar de maestros "Inexpertos". Biografías, trayectorias y práctica profesional. Revista Iberoamericana de Educación, 34(3), 1-11.

Avalos, B., Carlson, B., \& Aylwin, P. (2004). La inserción de profesores neófitos en el sistema educativo: ¿Cuánto sienten que saben y cómo perciben su capacidad docente en relación con las tareas de enseñanza asignadas? Informe proyecto Fondecyt 1020218.

Boerr, I. (2014). El largo camino de convertirse en profesor. Docencia, 54, 52-63.

Cisternas, T. (2011). Dificultades experimentadas por profesores principiantes de Educación Básica en sus prácticas de enseñanza y su relación con la Formación Inicial. CIDE, Universidad Alberto Hurtado, CEPPE. Recuperado desde: www.cide.cl/investigacion/investigacion_desarrollo_ detalle_ceppe.php. Recuperado enero 2015.

Cochran-Smith, M. (2004). Stayers, Leavers, Lovers, and Dreamers: Insight about teacher retention. Journal of Teacher Education, 55, 387-392.

Contreras, I., Vásquez, N., Rittershaussen, S., Solís, M. C., \& Núñez, C. (2013). La conducción de procesos de enseñanza aprendizaje de profesores novatos: ¿a mayores y mejores oportunidades de práctica en la formación inicial, mejores noveles de desempeño? Informe Proyecto Fondecyt 1100771.

Feiman-Nemser, S. (2000). From preparation to practice, designing a continuum to strengthen and sustain teaching. Teachers College Record, 103, 1013-1055.

Huberman, M., Gonauer, M.M., \& Marti, J. (1993). The Lives of Teachers. New York: Teachers College.

Marcelo, C. (1993). Cómo conocen los profesores la materia que enseñan. Algunas contribuciones de la investigación sobre conocimiento didáctico del contenido. En M.L. Montero, \& J.M Vez (Eds.), Las didácticas específicas en la formación del profesorado (pp. 151-186). Santiago: Tórculo

Marcelo, C. (Coord.). (2008). El profesorado principiante: Inserción a la docencia. Barcelona: OCTAEDRO

Marcelo, C. (2009) Los comienzos de la docencia: un profesorado con buenos principios. Revista de curriculum y formación del profesorado, 13(1).

Rodríguez, E. (2007). Las instituciones también cuentan. Los profesores principiantes en educación 
media frente a los desafíos del desarrollo profesional. Docencia, 33, 64-73.

St. Vincent, L., \& Gervais, C. (2010). ¿Está preparado el profesor novicio a enfrentar un problema ético en la escuela? Ponencia presentada en el II Congreso Internacional sobre Profesorado Principiante e Inserción Profesional a la Docencia. Recuperado desde: http://www.crifpe.ca/ conferences/view/77

Thompson, J. (2007). The first year teacher's survival guide. San Francisco: Jossey-Bass.

Veenman, S. (1984). Perceived problems of beginning teachers. Review of Educational Research, 54(2), 143-178. 